\title{
Publisher's Note: Coupling and braiding Majorana bound states in networks defined in two-dimensional electron gases with proximity-induced superconductivity \\ [Phys. Rev. B 96, 035444 (2017)]
}

Michael Hell, Karsten Flensberg, and Martin Leijnse

(Received 8 September 2017; published 12 September 2017)

DOI: 10.1103/PhysRevB.96.119906

This paper was published online on 31 July 2017 with an error in the title. The title should read as "Coupling and braiding Majorana bound states in networks defined in two-dimensional electron gases with proximity-induced superconductivity." The title has been corrected as of 11 September 2017. The title is incorrect in the printed version of the journal. 